\title{
Dysphagia as a Rare Presentation of Cervical Cancer with Mediastinal Metastasis
}

\author{
Vinit Singh ${ }^{1}$, Dhairya Gor ${ }^{2}$, Sarah Azad ${ }^{1}$, Anthony Ricca $^{1}$, Yiqing Xu ${ }^{3}$, Trishala Meghal ${ }^{4}$ \\ ${ }^{1}$ Department of Internal Medicine, Monmouth Medical Center, Long Branch, NJ, USA \\ ${ }^{2}$ Department of Internal Medicine, Jersey Shore University Medical Center, Neptune, NJ, USA \\ ${ }^{3}$ Division of Hematology and Oncology, Department of Internal Medicine, Maimonides Medical Center, Brooklyn, NY, USA \\ ${ }^{4}$ Division of Hematology and Oncology, Monmouth Medical Center, Long Branch, NJ, USA
}

Doi: 10.12890/2022_003136 - European Journal of Case Reports in Internal Medicine - ๑ EFIM 2022

Received: 19/12/2021

Accepted: 29/12/2021

Published: $24 / 01 / 2022$

How to cite this article: Singh V, Gor D, Azad S, Ricca A, Xu Y, Meghal T. Dysphagia as a rare presentation of cervical cancer with mediastinal metastasis. EJCRIM 2022;9: doi:10.12890/2022_003136.

Conflicts of Interests: The authors declare there are no competing interests.

This article is licensed under a Commons Attribution Non-Commercial 4.0 License

\section{ABSTRACT}

Dysphagia has a broad aetiology and so it is essential to identify the precise cause. Cervical cancer metastasis is distinctive in that it usually involves local lymph node invasion; however, approximately $1 \%$ of cases have mediastinal involvement, which can cause severe compressive symptoms in rare situations. To highlight an uncommon severe manifestation of cervical cancer relapse, we describe a case of dysphagia in a woman with a history of cervical cancer. After a thorough investigation that included endoscopy, endoscopy with ultrasound, an oesophagogram and fine needle aspiration, we eventually reached the diagnosis of mediastinal metastatic cervical cancer. Following interventions, the patient's condition gradually improved, both clinically and radiographically.

\section{LEARNING POINTS}

- Mediastinal lymph node enlargement causing dysphagia can be a presenting sign of a metastatic lesion from treated cervical cancer.

- A PEG tube is helpful in relieving dysphagia caused by metastatic mediastinal lymph node enlargement and as bridge for nutrition during chemotherapy.

- The VEGF inhibitor bevacizumab can be used in advanced metastatic cervical cancer.

- Check-point inhibitors like pembrolizumab can be used in metastatic cervical cancer.

\section{KEYWORDS}

Dysphagia, cervical cancer, metastasis, mediastinal lymph node, oesophagus

\section{INTRODUCTION}

Cervical cancer, although a largely preventable cancer with the human papillomavirus (HPV) vaccine, continues to impact women worldwide. The incidence of cervical cancer is highest in low- and middle-income countries and in medically underserved populations in more prosperous countries ${ }^{[1]}$. The management of cervical cancer involves multimodal treatment strategies, including chemotherapy, local radiation therapy and surgery. In addition, all patients who undergo curative therapy need periodic surveillance for disease progression or recurrence. The availability of multimodal treatment strategies and compliance with surveillance after treatment has led to better management of the disease with longer overall survival. The initial presentation of cervical cancer has been changing with a decrease in the proportion of patients with localized cervical cancer but a higher proportion with distant involvement, including regional and distant metastasis ${ }^{[2]}$. 
Cervical cancer metastases are often localized to the adjacent lymph nodes, but metastases to distant sites such as lymph nodes, bone and visceral organs have also been seen.

Here we report a case of dysphagia in a woman with a history of cervical cancer to highlight a rare presentation of cervical cancer relapse, and describe the investigational approach to rule out other possible pathologies for dysphagia. We also discuss available treatment strategies for metastatic cervical cancer.

\section{CASE DESCRIPTION}

A 49-year-old woman with a 2-year history of stage 3A squamous cell cervical cancer, treated with cisplatin and local high dose rate (HDR) brachytherapy, presented 9 months after the last follow-up, which included negative CT and PET scans. She presented to the emergency department (ED) with a 1-month history of increasing pain and progressive discomfort while swallowing. She felt discomfort while eating solid food but was able to tolerate liquids. Her dysphagia progressed to having a cough with the production of clear sputum. She was diagnosed with aspiration pneumonitis and discharged. However, she eventually developed dysphagia to both solids and liquids and was admitted for further evaluation. She reported a weight loss of around 20 pounds and denied any chest pain, neck mass or halitosis.

In the ED, physical examination was unremarkable except for fine crepitation on the right lung base with an Eastern Cooperative Oncology Group (ECOG) score of 0 . Initial laboratory results were essentially normal. CT of the chest with oral contrast demonstrated mild dilatation of the upper oesophagus with air-fluid levels, narrowing of the mid oesophagus, and collapse of the distal oesophagus. Also noted was a soft tissue prominence measuring $2 \times 2.3 \mathrm{~cm}$ immediately distal to a fluid-filled oesophageal segment. On CT, it was not clear if the mass was a mediastinal or endophytic oesophageal lesion. Also noted were a $2.0 \times 1.8 \mathrm{~cm}$ left supraclavicular lymph node (Virchow's node), patchy bilateral infiltrates at the lung bases, and a $12 \mathrm{~mm}$ left lower lung nodule. Upper $\mathrm{Gl}$ endoscopy showed severe stenosis of the oesophagus at $25 \mathrm{~cm}$ from the incisor with an intrinsic oesophageal diameter measuring $6 \mathrm{~mm}$ dilated afterwards to $12 \mathrm{~mm}$ by a through-the scope balloon dilator. Unfortunately, the endoscope could not transverse the stenotic area, and no further evaluation beyond the stricture was possible. On endoscopic ultrasound (EUS), several lymph nodes were visualized in the anterior mediastinum (level 6), measuring 7.2 mm, and at the aortopulmonary region (level 5), measuring $8.7 \mathrm{~mm}$. EUS-assisted fine needle aspirate (FNA) was obtained from the obstructing mediastinal mass. The patient further underwent an oesophagogram which showed a focal segment of severe narrowing within the mid oesophagus with moderate pre- stenotic oesophageal dilatation and retention of contrast because of delayed transit across the stenotic portion (Fig. 1). On histopathological assessment of the mediastinal mass aspirate, atypical cells were noted, positive for p40 on immunohistochemistry (IHC) consistent with the diagnosis of metastatic squamous cell cancer. The patient also underwent left supraclavicular lymph node biopsy, which was positive for both P16 and GATA3, confirming the metastatic squamous cell carcinoma diagnosis. The patient also underwent a whole-body PET-CT scan (Fig. 2), which showed increased uptake limited to the mediastinal lymph node and a left supraclavicular lymph node, also confirming the diagnosis of metastatic squamous cell carcinoma of the cervix causing oesophageal compression. There was no increased uptake in the lung nodule which suggested an inflammatory/infective process.
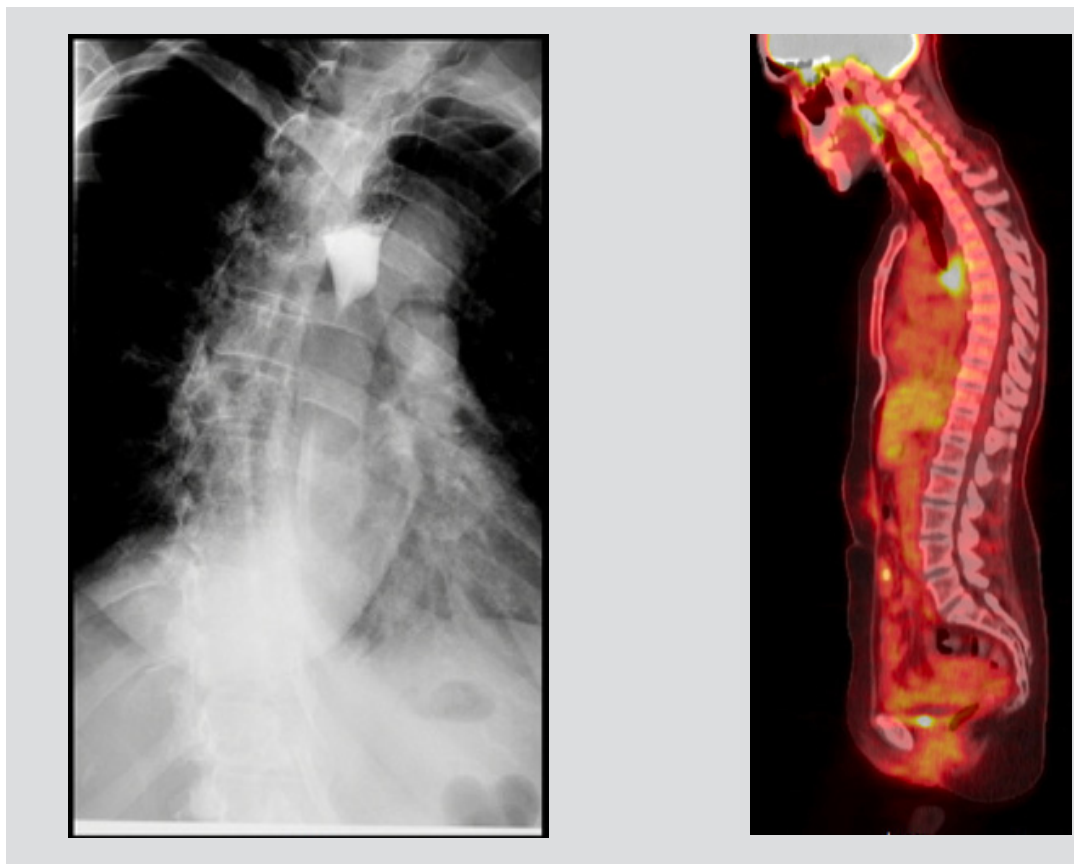

\begin{abstract}
Figure 1. A barium oesophagogram showing accumulation of contrast with severe narrowing in the mid oesophagus and prestenotic oesophageal dilatation
\end{abstract}

Figure 2. PET scan showing increased uptake limited to the mediastinal region 
The patient had a 1-month history of weight loss. After the swallow evaluation, she was started on a pureed diet. On the second day of admission, she had an increased cough with production of clear phlegm containing bits of food, so she was started on total parenteral nutrition. Later, a J-tube was placed to preserve the oesophagogastric junction's anatomic integrity should an oesophagectomy be needed. After 1 week of supplemental feeding, the patient was discharged from hospital and advised to follow up for a PET scan and treatment of relapsed cancer. The patient received standard of care treatment with carboplatin and paclitaxel in combination with bevacizumab. After the second cycle of chemotherapy, the symptoms of dysphagia had significantly improved and she was able to tolerate a regular diet.

Repeat CT scan findings after three cycles of chemotherapy showed improvement in metastatic disease with an interval decrease in the enlarged subcarinal lymph node from $1.8 \mathrm{~cm}$ to $1.5 \mathrm{~cm}$, decreased hilar lymphadenopathy, a decreased infiltrative tumour in the lung, and decreased size of the left supraclavicular lymph node from $2.5 \mathrm{~cm}$ at the time of diagnosis to less than $1 \mathrm{~cm}$. A CT scan also showed resolution of previously seen bilateral ground-glass opacity in the lungs, consistent with resolved aspiration pneumonitis.

\section{DISCUSSION}

The patterns of metastatic spread are unique to cervical cancer with $75 \%$ of cases present with local lymph node invasion. In distant metastases, the lung, liver and bone are the major sites involved ${ }^{[3]}$. Other rare sites for recurrence have also been reported, including the small bowel and a cyst in the hand ${ }^{[4]}$. There have been only a few reported cases of severe dysphagia caused by metastatic cervical cancer, all of which had mediastinal lymph node metastases as the primary cause of dysphagia ${ }^{[5,6]}$. Mediastinal metastases occur in approximately $1 \%$ of all cervical cancer cases. Our patient's case was complicated by the severity of dysphagia caused by mediastinal nodal compression. As part of the dysphagia evaluation, our patient underwent endoscopy and EUS. EUS-mediated FNA has been shown to be very useful in assessing mediastinal abnormalities and has a sensitivity and specificity of $69 \%$ and $100 \%$, respectively ${ }^{[7]}$. These patient's findings led to the final diagnosis of stage 4B squamous cell carcinoma.

After the diagnosis of cervical metastasis cancer was confirmed, the patient was started on carboplatin and paclitaxel with bevacizumab. Bevacizumab is a vascular endothelial growth factor (VEGF) inhibitor and was approved by the FDA for advanced cervical cancer in combination with carboplatin and paclitaxel in 2014. It has been shown to increase survival in advanced cervical cancer by around 3.4 months compared with carboplatin/paclitaxel alone ${ }^{[8]}$. In addition, patients with bevacizumab-containing therapy showed a better overall response, slower disease progression, and higher probability of response while maintaining the same quality of life ${ }^{[9]}$. However, monitoring in particular for grade 3 adverse effects such as thromboembolism and intestinal fistulas is necessary. VEGF is involved in various processes such as mitogenesis, angiogenesis, endothelial cell survival, and induction of haematopoiesis. Patients with high-grade cervical dysplasia and invasive carcinoma have increased expression of VEGF, which explains the response to bevacizumab ${ }^{[10]}$.

Apart from anti-angiogenic bevacizumab, FDA has also approved the PD-L1 inhibitor pembrolizumab for previously treated patients with recurrent or metastatic cervical cancer with disease progression on or after chemotherapy whose tumours express PD-L1 (CPS $\geq 1$ ) based on the results of the KEYNOTE-158 Trial (NCT02628067) ${ }^{[11]}$.

Cervical cancer continues to be a major burden of disease globally. Here we present a case of severe dysphagia due to mediastinal recurrence with excellent response to palliative chemotherapy with targeted therapy. Hence even in atypical presentations, it is essential to be highly suspicious for this diagnosis in order to ensure prompt treatment. Along with the targeted therapies, immunotherapy has created a ray of hope for further improvement in survival from this fatal disease. Hopefully, antibody-drug conjugates will further improve outcomes in cervical cancers.

\section{REFERENCES}

1. Cohen PA, Jhingran A, Oaknin A, Denny L. Cervical cancer. Lancet 2019;393:169-182.

2. Zhang Y, Guo Y, Zhou X, Wang X, Wang X. Prognosis for different patterns of distant metastases in patients with uterine cervical cancer: a population-based analysis. $J$ Cancer 2020;11(6):1532-1541.

3. Zhou S, Peng F. Patterns of metastases in cervical cancer: a population-based study. Int J Clin Exp Pathol 2020;13(7):1615-1623.

4. Bhandari V, Kausar M, Naik A, Batra M. Unusual metastasis from carcinoma cervix. J Obstet Gynecol India 2016;66(5):358-362

5. Hameed A, Dekovich AA, Lum PJ, Shafi MA. Dysphagia: an unusual presentation of metastatic uterine cervical carcinoma. J Coll Physicians Surg Pakistan 2017;27(3):187-188. Jahromi BM, Nhu QM, Moawad FJ. Metastatic cervical cancer causing esophageal compression and dysphagia. Clin Gastroenterol Hepatol $2019 ; 17(8): A 41-42$.

7. Devereaux BM, LeBlanc JK, Yousif E, Kesler K, Brooks JA, Mathur P, et al. Clinical utility of EUS-guided fine-needle aspiration of mediastinal masses in the absence of known pulmonary malignancy. Gastrointest Endosc 2002;56(3):397-401.

8. Tewari KS, Sill MW, Long HJ, Penson RT, Huang H, Ramondetta LM, et al. Improved survival with bevacizumab in advanced cervical cancer. N Engl J Med 2014;370(8):734-743.

9. Cella D. Bevacizumab and quality of life in advanced cervical cancer. Lancet Oncol 2015;16:241-243.

10. No JH, Jo H, Kim SH, Park IA, Kang D, Han SS, et al. Expression of vascular endothelial growth factor and hypoxia inducible factor-1a in cervical neoplasia. Ann N Y Acad Sci 2009;1171:105-110.

11. Chung HC, Ros W, Delord JP, Perets R, Italiano A, Shapira-Frommer R, et al. Efficacy and safety of pembrolizumab in previously treated advanced cervical cancer: results from the phase II KEYNOTE-158 study. J Clin Oncol 2019;37(17):1470-1478. 